\title{
A New Tariff Based Energy Saving and Sharing Scheme from Renewable Energy Using Smart Grid
}

\author{
V.Annapoorani ${ }^{\text {a, } 1}$, J. Banupriya ${ }^{\text {a }}$, P.Navaraja a ${ }^{\text {V.Chinnammal }}{ }^{\text {b }}$ \\ ${ }^{a}$ Assistant Professor, Department of ECE, Mahendra Institute of Technology \\ (Autonomous), Namakkal, Tamil Nadu, India \\ ${ }^{b}$ Assistant Professor (SS), Department of ECE, Rajalakshmi Institute of Technology, \\ Chennai, Tamil Nadu, India
}

\begin{abstract}
Abstract of our paper's major intent is to manage power and to share the solar load power to grid system by using smart grid technologies that is called as Demand Side Management (DSM). This paper gives the idea of modernized delivery system of electricity in which it observes, safeguards and adjusts accordingly with the energy that is used in home. The objective of the work is when the renewable resources are plentiful and electricity becomes affordable, time-of-use pricing, which allows customers to move some of their energy use to consistent and convenient moment of the day.
\end{abstract}

Keywords: Load power, grid system, DSM, renewable energy sources, Electricity, Arduino Uno Microcontroller.

\section{Introduction}

The entire load which is attached to the power grid varies outstandingly with respect to time. Even though the entire load is added up with the various separate options of the consumers, the whole load is not required to be either compulsorily stable or not moving faster. Considering the instance that a widespread TV schedule begins then million numbers of TV would start taking the current directly [1]. But conventionally a few free generators are pulled to be in a backup manner in order to acknowledge the usage of shoot up power. Thus, the smart grid might alert the TV sets or the customer to decrease the power for a shorter period or at regular intervals [2]. With the support of prediction algorithms found in the mathematics, it is easy to anticipate the number of backup generators utilized and also to obtain a definite failure rate. In the standard grid, the failure rate could be decreased only by the cost of the backup generators. In smart grid the decrease in load occurs even a little part of the customer is removed.

Though conventional load balancing techniques aimed to adapt consumers' spending habits in addition to making demand more uniform, advances in energy storage and individual renewable energy generation have made it possible to create sustainable power grids without impacting consumers' actions. Typically, saving energy during off-peak hours helps with peak-hour availability [3].

\footnotetext{
${ }^{1}$ V.Annapoorani, Assistant Professor, Department of ECE, Mahendra Institute of Technology (Autonomous), Namakkal, TN, India, Email: annapooranisathya@gmail.com.
} 


\section{Condition 1: Hospital}

The most important is to take care of human life, so 24 hours no limits of solar power, in demand time period.

\section{Condition 2: Industrial}

Generally, EB board provides a power of KVA based on equipment if the solar power demand occurs of about 50 \%rapidly the total solar power will be stopped automatically. When the generating solar power increases or reaches $75 \%$ automatically total power enters into the industries [4]. Every time total solar power is calculated and monitors the wastage of power. Any one of the industries that involves power wastage, the CT is monitoring and intimating the power wastage period of few times and thus minimizes the load. Since the loads are not minimized automatically total solar powers are shutdown [5].

\section{Condition 3: Domestic}

Generally, EB board providing a single phase or three phase power supply based on appliance and if the solar power demand occurs 75 percentages suddenly the total solar power is stopped automatically and at the same time solar power engaging the demand period when generating the solar power increasing or reaching 95 percentages, automatically total solar power enters into the domestic. Each and every time total solar power is calculated and monitored the power wastage and any one domestic involved power wastage the $\mathrm{CT}$ are monitoring and intimating the power wastage periods of few times, suddenly minimizing the load. Because the loads are not minimized automatically total solar powers are shutdown [7].

\section{Demand Switches}

In this system, the two demand switches are used. Here, the toggle type of demand switch is used. These switches are interfaced with the Arduino Uno microcontroller such that when demand occurs the toggle switch starts to operate automatically [8].

\section{Buzzer}

To indicate the wastage of power a buzzer is used. When the solar power consumption exceeds the prescribed level, the power wastage buzzer provides an indication in order to trip the circuit.

\section{Node MCU Unit}

A node MCU unit provides the connection between the IOT and the working model is shown in Figure 1.

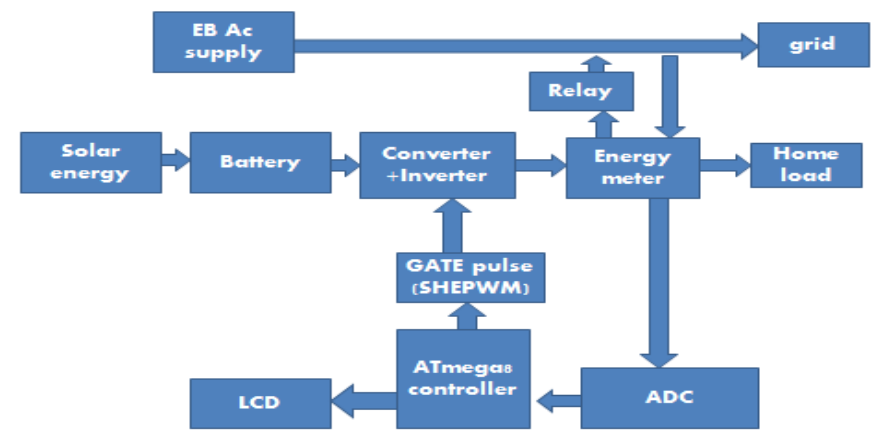

Figure 1. Block Diagram of Proposed System 


\section{Description}

The ATmega16 is an AVR RISC-based energy saving Complementary Metal Oxide Semiconductor eight-digit microcomputer. For every time period, the outputs of the microcomputer reach one MIPS/MHz with the help of strong commands of solar energy. Thus, this makes the designers to have an equal match of energy usage and time periods. A thirty-two universal storage elements and huge instruction set is present in automatic voltage regulator. A part of central processing unit that performs calculations and logical operations is in straightaway contact with every thirty-two storage elements. This makes the 2 storage elements to be approached every clock cycle with a single instruction [9]. As a result, the architecture is more code effective than traditional CISC microcontrollers. The Atmel ATmega16 is a solar-powered microcontroller that can handle a wide range of embedded control applications is shown in Figure 2.

\section{Smart Grid and Micro Grid}

This is related with the upcoming peer groups of electrical system which has an enhancing power saving structure. With the addition of electrical network and IT technology, it interchanges the actual power demanded/ power system data two-way joining the vendor and the buyer [10]. But the buyer part has a smaller sized grid and different factors like energy control, networking etc. which makes others to make up the micro grid. Smart meters, solar panels for the home, slick machines, motor car charging are examples of electric products. Slight distributed style energy storage systems are deployed in the micro grid, and they help to improve smart grid performance by reacting quickly to real-time electricity demand/supply data.

\section{Energy Storage System (ESS)}

Renewable energy generated electrically is stored in a storage system for further use. Based on ESS Capacity, ESS products are used. They are categorized as three groups: ESS has given as megawatt (MW) level in case of electricity frequency adjustment. The next level of ESS is large capacity (MW) used for coping with demand at peak times of electricity, and final level of ESS is medium capacity kilowatt (kW) used for domestic use.

\section{Relay}

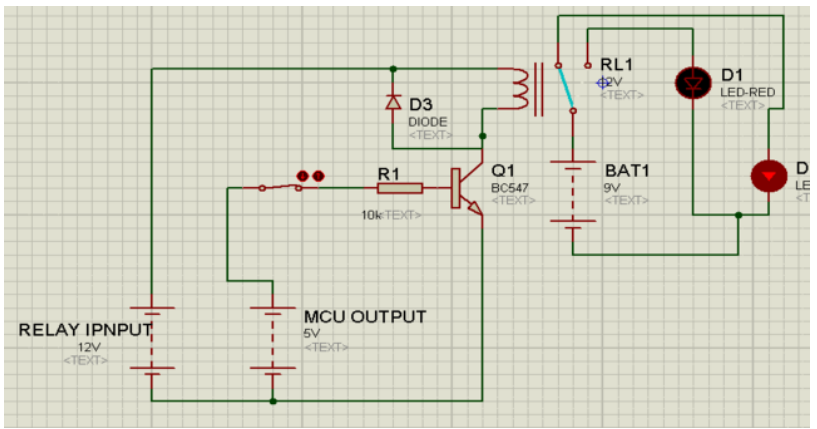

Figure 2. Circuit diagram of Relay

\section{Working of Relay}

A relay is a switch which operates electrically so that it attracts lever and changes the position of switch. Magnetic field is generated by current flowing through the coil which is attracted by lever and changes the switch contacts. So current in the coil is in on or off position. This can be double thrown (change over) switches. 
The operation of Relays shows that one circuit is turned on whereas the second circuit is disconnected. For example, $230 \mathrm{~V}$ AC main used as relay to switch a $230 \mathrm{~V}$ AC mains circuit. Due to maximum output of 555 timer IC which has current of $200 \mathrm{~mA}$, so these devices can act as relay coils directly without amplification. Relays are usually SPDT or DPDT

\section{IOT WIFI ESP8266}

WIFI module Wi-Fi ESP8266 is a low-cost chip with TCP/IP stack and microcontroller is shown in Figure 3. In our paper, we use a Web server to display information such as the amount of energy consumed in units, the bill, and if any theft occurs. As a result, any user can access the information from anywhere in the world. The paper's information is displayed on the Thing speak web page.

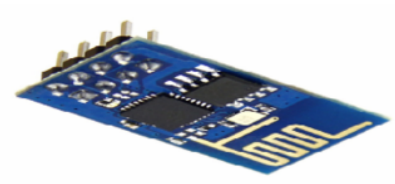

Figure 3. Diagram of WIFI

\section{Block Diagram of Solar Power Supply}

The safe operating area of a transistor is defined by minimizing available output current for large input to output voltage differentials. These regulators are economical and easy to use, which is suitable to design a system with bountiful printed-circuit boards by which unregulated dc is brought to each board and regulation is performed is shown in Figure 4.

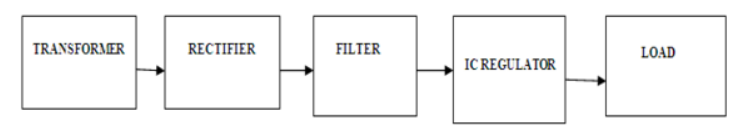

Figure 4. Power Supply Block Diagram

\section{Bridge Rectifier Circuit}

The circuit shows as a bridge rectifier which contains four diodes are connected as shown in the Figure 5 . The input to the circuit is given diagonally opposite sides and the output is taken between other two corners. It produces twice that of output voltage as compared to full wave rectifier. Main advantage of bridge rectifier is it produces two times of output voltage over a conventional full-wave rectifier.

\section{LCD Display}

LCD stands for Liquid Crystal Display which has limited viewing angle is shown in Figure 5. LCD used as economical one in order to display 16 characters and symbols. D0-D7 will represent as commands or as data on logic states. RS: RS $=1$ for this status Bits D0 - D7 used for representing addresses of characters to be displayed. Some Built in processor addresses has built in "map of characters" and to show appropriate 
symbols. RS $=0$ for this status Bits D0 - D7 are commands which is used to find the display mode is shown in Figure 6.

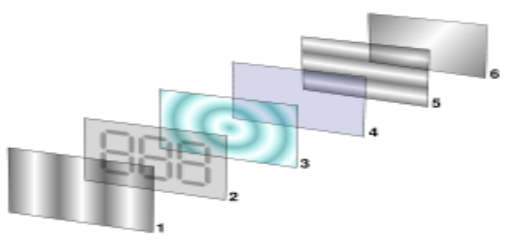

Figure 5. Structure of LCD Display

\section{Features of LCD Display}

- $\quad$ Cursor has $5 \times 8$ dots

- $\quad$ Built in integrated controller (ks 0066 or equivalent)

- $\quad$ Operates power $+5 \mathrm{v}$

\section{Schematic Diagram of LCD Display}

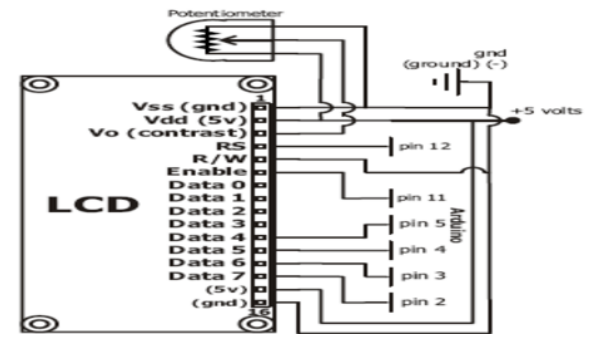

Figure 6. Schematic diagram of LCD Display

\section{Operation}

When demand occurs on the grid side the demand switch 1(toggle switch) will operates. The priority is given to the hospital and industrial concern. So that the load from the domestic side is shared to the industrial and hospital facilities. At the same time the minimum amount of supply is given to the domestic side at that situation if they consume too much of supply then the power wastage buzzer will give intimation and then trips the circuit. If the demand on the grid side is further increased then the demand switch 2 will operate, so that the load from the industrial side is shared to hospital facilities. At that situation if the industry consumes power wastage then the power wastage buzzer will provide intimation and then trips the circuit.

Here we are using toggle type demand switch. The demand switch operation depends on the fixed rating. The current transformer continuously senses the current rating and if there is any excess current then the current transformer will sense the signal and gives the signal to the relay circuit which trips the circuit accordingly. The IOT module continuously monitors the overall operation of the system. 


\section{Result and implementation}

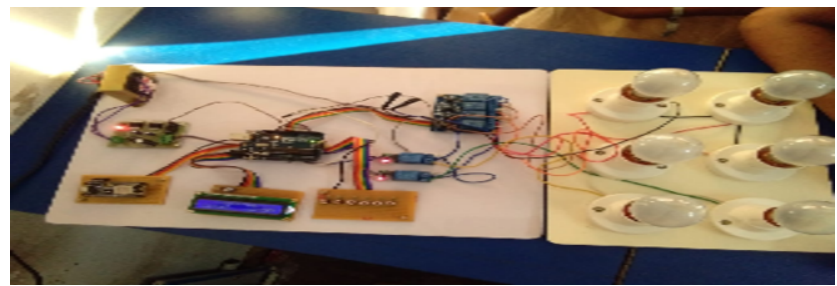

Figure 7. Solar based power sharing

Apart from the regular monitoring of the solar power line, it also helps to monitor the weather and its effect on the solar power system is shown in Figure 7. With this idea about the weather patterns [7] near the solar power transmission line is obtained and also which event is going to occur based on the data collection is predicted shown in Figure 8. Also, in India the problem of solar power theft is common, thus this problem is avoided with the help of IOT and prevents the solar power theft.

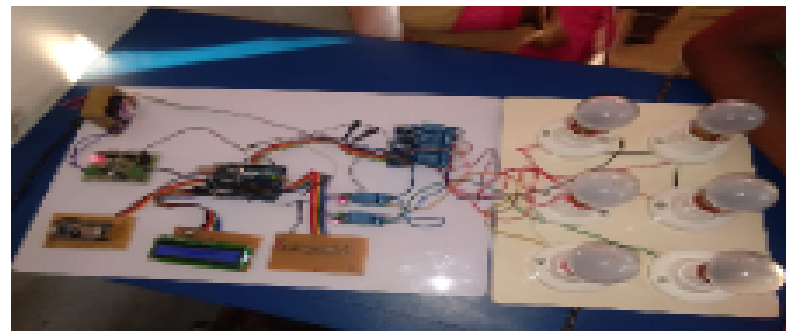

Figure 8. Diagram for Smart mode

\section{Conclusion}

Thus, we can conclude that this work using smart grid solves the problem of unidirectional information flow, wastage of energy and manages the growing energy demand and provides intelligent and advanced power control using AVR Studio software and can be applied in three different modes as home mode, Grid mode and smart mode. Hence the tariff can be reduced.

\section{References}

[1] Yaacoub, E., \& Abu-Dayya, A. (2014). Automatic meter reading in the smart grid using contention based random access over the free cellular spectrum. Computer Networks, 59, 171-183. https://doi.org/10.1016/j.bjp.2013.10.009

[2] Sun, H., Nallanathan, A., Tan, B., Thompson, J., Jiang, J., \& Poor, H. (2012). Relaying technologies for smart grid communications. IEEE Wireless Communications, 19(6), 52-59. https://doi.org/10.1109/MWC.2012.6393518

[3] Yigit, M., Gungor, V. C., \& Baktir, S. (2014, September 9). Cloud Computing for Smart Grid applications. Computer Networks. Elsevier B.V. https://doi.org/10.1016/j.comnet.2014.06.007

[4] Deng, R., Yang, Z., Chow, M. Y., \& Chen, J. (2015). A survey on demand response in smart grids: Mathematical models and approaches. IEEE Transactions on Industrial Informatics, 11(3), 570-582. https://doi.org/10.1109/TII.2015.2414719 
[5] Ma, R., Chen, H. H., Huang, Y. R., \& Meng, W. (2013). Smart grid communication: Its challenges and opportunities. IEEE Transactions on Smart Grid, 4(1), 36-46. https://doi.org/10.1109/TSG.2012.2225851

[6] Bush, S. F. (2014). Network theory and smart grid distribution automation. IEEE Journal on Selected Areas in Communications, 32(7), 1451-1459. https://doi.org/10.1109/JSAC.2014.2332132

[7] Collier, S. E. (2017, March 1). The Emerging Enernet: Convergence of the Smart Grid with the Internet of Things. IEEE Industry Applications Magazine. Institute of Electrical and Electronics Engineers Inc. https://doi.org/10.1109/MIAS.2016.2600737

[8] Temel, S., Gungor, V. C., \& Koçak, T. (2014). Routing protocol design guidelines for smart grid environments. Computer Networks, 60, 160-170. https://doi.org/10.1016/j.bjp.2013.11.009

[9] Meng, W., Ma, R., \& Chen, H. H. (2014). Smart grid neighborhood area networks: A survey. IEEE Network, 28(1), 24-32. https://doi.org/10.1109/MNET.2014.6724103

[10] Wang, W., Xu, Y., \& Khanna, M. (2011, October 27). A survey on the communication architectures in smart grid. Computer Networks. Elsevier B.V. https://doi.org/10.1016/j.comnet.2011.07.010

[11] Hannoon, N., Vijayakumar, V., Vengatesan, K., \& Hidayat, N. (2019). Small Signal Fault Analysis for Renewable Energy (Wind) Power System Distributed Generation by Using MATLAB Software (Simulink). Journal of Computational and Theoretical Nanoscience, 16(2), 537-543. 